Psychotherapeut 2010 · 55:453-454 DOI 10.1007/s00278-010-0785-0

Online publiziert: 28. Oktober 2010

(c) Springer-Verlag 2010

\author{
Bernhard Strau $\AA^{1} \cdot$ Harald J. Freyberger ${ }^{2}$ \\ ${ }^{1}$ Institut für Psychosoziale Medizin und Psychotherapie, Universitätsklinikum, \\ Friedrich-Schiller-Universität Jena \\ ${ }^{2}$ Klinik und Poliklinik für Psychiatrie und Psychotherapie, Universität Greifswald \\ am HANSE-Klinikum Stralsund
}

\title{
Supervision als zentraler Bestandteil professioneller Psychotherapie
}

und klinischer Expertise, also der Professionalisierung, Rollenfindung und der Weiterentwicklung professioneller Identität, auch im Sinne lebenslangen Lernens, der Qualitätskontrolle, der Sicherstellung von „Manualtreue“ bzw. korrekter Anwendung erlernter Interventionen, der Problemlösung bzw. -bewältigung, aber auch der Psychohygiene, im Sinne einer professionellen Unterstützung und Burnout-Prophylaxe. In der sog. institutionellen Supervision werden darüber hinausgehend Konzepte, Strukturen und Prozesse durch von außen kommende Experten in Einrichtungen verschiedener Art einem kritischen Betrachtungsprozess unterzogen.

Es ist über zehn Jahre her, seit in der Zeitschrift Psychotherapeut das Thema Supervision im Rahmen einer Übersichtsarbeit behandelt wurde (Auckenthaler 1999). Angesichts der großen Bedeutung von Supervision in der Ausbildung und in der Praxis von Psychotherapeuten ist es angemessen, einmal wieder Themen im Zusammenhang mit klinischer Supervision zusammengefasst darzustellen. Darüber hinaus ist die Supervision selbst im vergangenen Jahrzehnt Gegenstand eines Institutionalisierungsprozesses verschiedener Fachgesellschaften geworden, den es durchaus kritisch zu betrachten gilt.

In diesem Schwerpunktheft wird zunächst in einer Übersicht beschrieben, welche Fragen zur klinischen Supervision die Psychotherapieforschung bisher beantworten kann. Dies sind noch nicht viele, dennoch gibt es inzwischen klarere Hinweise dafür, dass Supervision „wirksam“ ist. Innerhalb des „Forschungsgutachtens zur Ausbildung von psychologischen Psychotherapeuten und Kinderund Jugendlichenpsychotherapeuten", in dessen Kontext zahlreiche Befragungen durchgeführt wurden, wurden vielfältige Informationen speziell zur Supervision gesammelt, die von Nodop et al. zusammengefasst werden. Hier bleibt festzuhalten, dass Supervision von den Befragten als ein überaus positiv bewerteter, zentraler Baustein ihrer Ausbildung aufgefasst wird.

Für die empirische Forschung zur Supervision sind spezifische Methoden notwendig. Ein Ansatz hierfür wird mit der Verhaltenstherapie-Kompetenz-Checkliste in dem Beitrag von Linden u. Langhoff vorgestellt. Ein kontextanalytisches Konzept der Supervision, aus dem sich auch eine qualitative Forschungsmethode ableiten lässt, wird in dem Text von KahlPopp beschrieben. Riessen et al. schließlich machen deutlich, wie supervisorische Arbeit die Möglichkeit bietet, auch in der manualisierten Verhaltenstherapie viele praxisnahe Erfahrungen zu sammeln.

Der Themenschwerpunkt wird mit einer Diskussion der Frage nach der Professionalisierung von Supervision abgerundet, in deren Rahmen eine vergleichende Bewertung verschiedener Trainings- und Ausbildungskonzepte erfolgt.
In der klinischen Praxis dient Supervision einerseits der Konsolidierung klinischer Erfahrung, klinischen Wissens 


\section{Korrespondenzadresse}

\section{Prof. Dr. Bernhard Strauß}

Institut für Psychosoziale Medizin und

Psychotherapie, Universitätsklinikum,

Friedrich-Schiller-Universität Jena

Stoystr. 3, 07740 Jena

Bernhard.Strauss@med.uni-jena.de

\section{Literatur}

Auckenthaler A (1999) Supervision von Psychotherapie: Behauptungen - Fakten -Trends. Psychotherapeut 44:139-152

Rappe-Giesecke K (Hrsg) (2009) Supervision für Gruppen und Teams, 4. Aufl. Springer, Berlin Heidelberg New York Tokio

\section{Schwerpunktthemen}

Das Herausgebergremium der Zeitschrift Psychotherapeut lädt Autorinnen und Autoren ein, an den geplanten Schwerpunkten mitzuarbeiten und geeignete Manuskripte einzureichen. Diese werden dem üblichen Reviewverfahren unterzogen. Bitte schicken Sie Ihren Beitrag an die Redaktion:

Regine.Karcher-Reiners@springer.com

Die Schwerpunktplanung ist vorläufig und kann kurzfristig umdisponiert werden.

Heft 3/11: Chronische Erschöpfung (Deadline 14.1.2011)

Heft 4/11: Gruppenpsychotherapie (Deadline 18.3.2011)

Heft 5/11: Psychoonkologie (Deadline 25.5.2011)

Heft 6/11: Psychotherapie und elektronische Medien (Deadline 26.7.2011)

Heft 1/12: Migration (Deadline: 17.9.2011)

Heft 2/12: Themata der Lindauer Psychotherapiewochen 2011 (Deadline 18.11.2011)

Heft 3/12: Neurowissenschaften (Deadline 14.1.2012) 